\title{
Über neue jodometrische Methoden, die auf der Bildung und Messung von Jodcyanid beruhen. I.
}

\author{
Von Rudolf Lang.
}

I. Allgemeines.

Nach E. v. MEYer ${ }^{1}$ ) führt dje Einwirkung von Jod auf Cyanwasserstoff in wäBriger Lösung zu dem Gleichgewicht

$$
\mathrm{J}_{2}+\mathrm{HCN} \rightleftharpoons \mathrm{JCN}+\mathrm{H}^{*}+\mathrm{J}^{\prime}
$$

Eigene Versuche überzeugten mich, daß Jod in mineralsaurer Lösung bei Anwesenheit von Cyanwasserstoff und starken Oxydationsmitteln wie Nitrit, Permanganat, Bromat, Jodat und Perjodat, quantitativ in Jodcyanid übergeht. Dies ist einfach zu erklären. Denn das nach (1) gebildete Jodid reagiert in Gegenwart von oxydierenden Stoffen nach

$$
J^{\prime}+(\cdot)=J
$$

weiter, während demzufolge die Reaktion (1) von links nach rechts fortschreitet. Es werden daher beide Vorgänge, von denen jeder die Folgereaktion des anderen ist, solange verlaufen, bis schlieBlich das gesamte Jod zu Jodeyanid oxydiert ist. Durch Summation von (1) und (2) ergibt sich dementsprechend das Schema

$$
\mathrm{J}+\mathrm{HCN}+{ }^{\cdot}=\mathrm{JCN}+\mathrm{H},
$$

wonach die Oxydation von Jod zu Jodcyanid erfolgt. Selbstverständlich läßt sich auch Jodid nach

$$
\mathrm{J}^{\prime}+\mathrm{HCN}+2^{(\cdot)}=\mathrm{JCN}+\mathrm{H}^{*}
$$

zu Jodcyanid oxydieren.

Man kann nun diese Reaktionen maßanalytisch zur Bestimmung von Jod und Jodid verwerten. Denn die Entladung von Jodion erfolgt in mineralsaurer Lösung durch Jodat oder Permanganat sehr rasch und der Endpunkt der Oxydation läßt sich in Gegenwart von Cyanwasserstoff mit aller Schärfe an dem Verschwinden der Jod-

1) Journ. prakt. Chem. 36 (1887), 292. 
stärkereaktion erkennen. Daß hier Stärke als Indikator verwendbar ist, zejgt eine Betrachtung der Konstante des Gleichgewichtes (1), die von $\mathrm{L}$. KovACH${ }^{1}$ ) $\mathrm{zu} \frac{[\mathrm{HCN}] \cdot\left[\mathrm{J}_{2}\right]}{[\mathrm{JCN}] \cdot\left[\mathrm{H}^{\prime}\right] \cdot\left[\mathrm{J}^{\prime}\right]}=1,37$ in saurer Lösung ermittelt wurde. Gegen Ende der Oxydation haben [HCN], [JCN] und $\left[\mathrm{H}^{\prime}\right]$ endliche Werte. Ist daher $\left[\mathrm{J}^{\prime}\right]$ sehr klein oder null geworden, so gilt dies auch von $\left[\mathrm{J}_{2}\right]$ und umgekehrt. Mit dem Ende der Oxydation verschwinden daher gleichzeitig $\left[J_{2}\right]$ und $\left[J^{\prime}\right]$ und die Bedingungen für das Bestehen der Jodstärkereaktion hören auf. Jodcyanid allein färbt Stärke nicht. Hier und da wird in der Literatur zwar das Gegenteil behauptet, aber zu Unrecht. Denn es ist eben neben Jodeyanid die Anwesenheit mindestens einer Spur Jodid erforderlich, damit die Jodstärkereaktion auftreten kann. ${ }^{2}$ )

Die Messung des Jods oder Jodids kann man nun in doppelter Art durchführen. Einmal kann man mit dem Oxydationsmittel als MaBflüssigkeit titrieren; dann aber kann das Oxydationsmittel im Ubberschuß zugesetzt werden. Nach Entfernung des letzteren ist, das gebildete Jodcyanid mit Thiosulfat ${ }^{3}$ ) gemäß der Gleichung

$$
\mathrm{JCN}+2 \mathrm{~S}_{2} \mathrm{O}_{3}{ }^{\prime \prime}+\mathrm{H}^{\prime}=\mathrm{J}^{\prime}+\mathrm{HCN}+\mathrm{S}_{4} \mathrm{O}_{8}{ }^{\prime \prime}
$$

titrierbar. Schließlich kann man beide Methoden hintereinander an. wenden, wobei die eine zur Kontrolle der anderen dienen kann.

In dem besonderen Falle, daß zur Oxydation des Jodids Jodat oder Perjodat verwendet wird, tritt auch das gesamte, den beiden letzteren entstammende Jod zu Ende der Reaktion als Jodcyanid auf. Das allgemeine Schema, nach dera Jodat in den meisten Fällen oxydierend wirkt, ist

$$
\mathrm{JO}_{3}{ }^{\prime}+6 \mathrm{H}^{\prime}=\mathrm{J}+3 \mathrm{H}_{2} \mathrm{O}+5^{(\cdot)} \text {. }
$$

Substituiert man hierin für $\mathrm{J}$ den Wert aus $(3)\left(\mathrm{J}=\mathrm{JCN}+\mathrm{H}^{*}\right.$ - HCN - (.) ), so erhält man das allgemeine Schema für die Oxydationswirkung von Jodat in Gegenwart von Cyanwasserstoff:

$$
\mathrm{JO}_{3}{ }^{\prime}+\mathrm{HCN}+5 \mathrm{H}^{\prime}=\mathrm{JCN}+3 \mathrm{H}_{2} \mathrm{O}+4^{(\cdot)}
$$

oder bei Perjodat

$$
\mathrm{JO}_{4}{ }^{\prime}+\mathrm{HCN}+7 \mathrm{H}^{\circ}=\mathrm{JCN}+4 \mathrm{H}_{2} \mathrm{O}+6^{(\cdot)} \text {. }
$$

Bei (5) findet also eine Reduktion des Jods urn 4, bei (6) eine solcho

1) Z. physik. Chem. 80 (1912), 107.

2) Uber die Empfindlichkeit der Jodstärkereaktion in diesem Falle vgl. die Ausführungen S. 337.

i) MoLnkxE, Z. anorg. Ohem. 2 (1892), 157. 
um 6 Oxydationsstufen statt, entsprechend der Tatsache, daß das Jod im Jodat und Perjodat positiv 5- bzw. positiv 7wertig im Jodeyanid dagegen positiv 1 wertig ${ }^{1}$ ) aufzufassen ist. Da diese Reduktion aber stets über freies Jod führt, kann man ganz allgemein mit Jodat oder Perjodat oxydierbare Stoffe bei Anwesenheit von Cyanwasseratoff bis zum Verschwinden der Jodstärkereaktion titrieren und auch das gebildete Jodcyanid mit Thiosulfat messen. Die Oxydation kann sowohl in salzsaurer als auch schwefelsaurer Lösung vorgenommen werden. Bedingung für eine direkte Titration mit Jodat ist aber, daB die Folgereaktionen (1) und (2) nicht rascher ablaufen als der eigentliche, primäre Oxydationsvorgang, da dann der Endpunkt der Oxydation nicht durch das Verschwinden der Jodstärkereaktion angezeigt würde; letztere braucht unter Umständen gar nicht aufzutreten. Man kann in solchen Fällen aber stets mit überschüssigem Jodat oxydieren und den Überschuß in einfacher Weise zurücktitrieren, wie in den weiteren Abschnitten gezeigt werden wird.

Jodat wurde schon vor längerer Zeit von ANDREws') zu oxydimetrischen Bestimmungen allgemein empfohlen. Seine Methode beruht darauf, daB bei der Oxydation mit Jodat in stark salzsaurer Lösung das gesamte, aus dem Jodat frei werdende, Jod am Ende der Reaktion in Jodmonochlorid übergeführt ist, was man an Verschwinden der Violettfärbung von zugefügtem Chloroform erkennt. Andrews arbeitet stets in stark salzsaurer, $12-15 \% \mathrm{HCl}$ enthaltender Jösung, so daß bei seiner Methode fast ausschließlich die Wirkung des aus dem Jodat mit Salzsäure gebildeten Chlors und Jodmonochlorids zur Geltung kommt. Dagegen benützen die hier beschriebenen Jodatmethoden, bei welchen eine Chlorwasserstoffkonzentration von 3-4\% genügt, nebst der intermediären Wirkung des Chlors auch die direkte Oxydationswirkung des Jodats, wie beim Arbeiten in schwefelsaurer Lösung. Die geänderten Arbeitsbedingungen ermöglichen die Anwendbarkeit der neven Methoden in Fällen, wo ANDREws' Verfahren versagt, so bei der Bestimmung von Bromid, von Jodid, Bromid und Chlorid nebeneinander $\left.{ }^{3}\right)$; ferner bei der Bestimmung von arseniger oder antimoniger Säure neben Ferroeisen. Win Vorteil ist auch die Anwendbarkeit von Stärke als Indikator. Wie schon oben erwähnt, ist das am Ende der Titration mit Jodat gebildete

ग) Vgl. Abeqa, Handbuch d. anorg. Chemie IV, 2, 276.

2) Z. anorg. Chem. 36 (1903), 76.

$\left.{ }^{3}\right)$ Uther die Bestimmung von Bromid sowie der drei Halogenide nebeneinander wird gesondert berichtet werden. 
Jodcyanid ohne weiteres mit Thiosulfat meBbar. Analog wäre das bei ANDREws' Methode gebildete Jodmonochlorid erst nach Zusatz von Jodkalium titrierbar. Soweit mir bekannt ist, wurde bisher noh nicht versucht, diese Möglichkeit analytisch zu verwerten.

\section{Bestimmung von Jod in Jodiden.}

Methode A.

Die Lösung des Jodids wird in einem lang- und enghalsigen Kolben') mit dem gleichen Volumen, mindestens aber rnit $50 \mathrm{ccm}$ 2,5 fach normaler Salz- oder Schwefelsäure angesänert, 6- $8 \mathrm{~cm}$ einer $1 / 2 n$-Kaliumcyanid- und etwas Stärkelösung hinzugefügt und darm 2-3 g Harnstoff eingetragen. Nun wird unter Umschwenken des Kolbens eine $1 / 2$ mol-Natriumnitritlösung hinzugetropft, bis die anfangs auftretende Blaufärbung wieder verschwunden ist. Nach 10 bis 15 Minuten wird mit 1/10 n-Thiosulfatlösung titriert. Die Anwesenheit von Bromiden, Chloriden und Nitraten stört nicht. In Gegenwart von Bromid färbt sich die Lösung gegen Ende der Oxydation infolge der Bildung von Jodbromid rotbraun. Letzteres geht bald in farbloses Jodeyanid über, wenn die Oxydation beendet ist.

Belegana'ysen:

16,5988 g reines Kaliumjodid wurden zu 21 gelöst und ron dieser Lösung clurch Ablassen aus einer Bürette abgemessene Mengen nach der beschriebenen Methode titriert. Die verwendete Thiosulfatlösung war mit reinem Jod eingestellt.

\begin{tabular}{|c|c|c|c|c|c|c|c|c|}
\hline \multirow{2}{*}{\multicolumn{2}{|c|}{$\begin{array}{l}\text { Angewendet ccm } \\
\text { Verbraucht }\end{array}$}} & & \multicolumn{6}{|c|}{ mit $\mathrm{H}_{2} \mathrm{SO}_{4}$ angesäuert } \\
\hline & & $\mathrm{KJ} . \cdots$ & & 20,07 & & 30,12 & 40,20 & \\
\hline \multirow{3}{*}{$\begin{array}{l}\text { Verbraucht } \\
\text { Berechnet }\end{array}$} & $"$ & $1 / 10 \mathrm{II}-\mathrm{Na}_{2} \mathrm{~S}_{2} \mathrm{O}_{3}$ & & & & & & \\
\hline & & $"$ & & 20 & & & 40,19 & \\
\hline & & & \multicolumn{4}{|c|}{ mit $\mathrm{HCI}$ angesäuert } & & \\
\hline \multirow{4}{*}{$\begin{array}{l}\text { Angewendet } \\
\text { Verbraucht } \\
\text { Berechnet }\end{array}$} & & & & & & & \multirow{3}{*}{\multicolumn{2}{|c|}{$\begin{array}{l}\text { bei jedem Ver- } \\
\text { suche waren } \\
0,5 \mathrm{~g} \mathrm{KBr} \text { an- } \\
\text { wesend }\end{array}$}} \\
\hline & , & & & & & & & \\
\hline & $"$ & & & 20 , & 25 & & & \\
\hline & & & \multicolumn{4}{|c|}{ mit $\mathrm{HCl}$ anges: } & & \\
\hline & & & & & & & \multirow{3}{*}{\multicolumn{2}{|c|}{$\begin{array}{l}\text { bei jedem Ver- } \\
\text { suche waren } \\
0,5 \mathrm{~g} \mathrm{NH}_{4} \mathrm{NO}_{3}\end{array}$}} \\
\hline & $"$ & $1 / 10 \mathrm{n}-\mathrm{Na}_{2} \mathrm{~S}_{2} \mathrm{O}_{3}$ & & & & & & \\
\hline & $"$ & & & 20 & 25,0 & 30,04 & & \\
\hline
\end{tabular}

Es wurden also durchwegs der Theorie entsprechende Werte erhalten, da die Versuchsfehlergrenze nirgends überschritten wurde.

1) Bei Anwendung solcher Gefäße wird der Analytiker und seine Umgebung durch den sich entwickelnden Cyanwasserstoff nicht im geringsten belästigt oder gefährdet. 
Methode B.

Die Lösung des Jodids wird mit dem gleichen Volumen, mindestens aber mit $50 \mathrm{ccm}$ 2,5 fach n-Salzsäure oder 4-5fach nSchwefelsäure angesäuert, $6-8 \mathrm{~cm} 1 / 2 \mathrm{n}$-Kaliumcyanid- und etwas Stärkelösung hinzugefügt und mit $1 / 40 \mathrm{~mol}$.-Kaliumjodatlösung bis zuin Verschwinden der anfangs auftretenden Jodstärkereaktion titriert. Zur Kontrolle kann man dann noch das gebildete Jodcyanid mit Thiosulfat titrieren. Die Methode ist in Gegenwart von Bromiden, Chloriden und Nitraten anwendbar.

Beleganalysen: mit $\mathrm{HCl}$ angesäuert

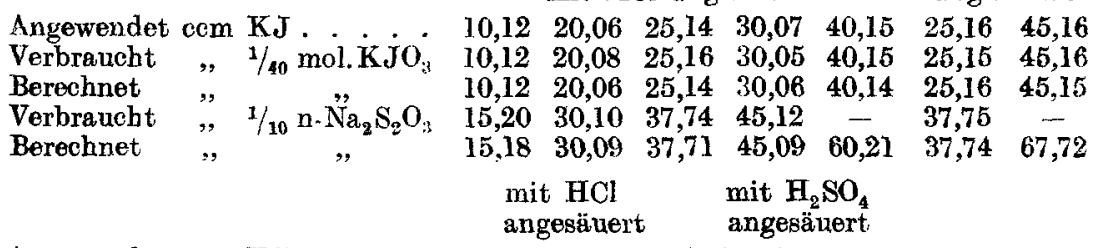

Angewendet $\mathrm{cm} \mathrm{KJ} . . \quad . \quad . \quad 10,07 \quad 20,11 \quad 30,04 \quad 25,06 \quad 30,10$ bei jedem Ver-

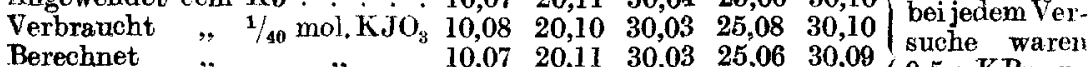

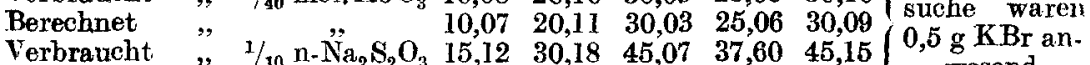
$\begin{array}{cccccccc}\text { Berechnet " } & 1 / 10 & \mathrm{n}-\mathrm{Na}_{2} \mathrm{~S}_{2} \mathrm{O}_{3} & 15,12 & 30,18 & 45,04 & 37,60 & 45,15 \\ & & 15,10 & 30,16 & \mathbf{4 5 , 0 4} & 37,59 & 45,13\end{array}$ wesend $\begin{array}{cl}\text { mit } \mathrm{HCl} & \text { mit } \mathrm{H}_{2} \mathrm{SO}_{4} \\ \text { angesäuert } & \text { angesänert }\end{array}$

Angewendet cem KJ . . . . . 10,05 20,08 $30,10 \quad 25,06 \quad 30,05$ Verbraucht " $1 / 40$ mol.KJO $10,06 \quad 20,08 \quad 30,11 \quad 25,06 \quad 30,06$ $\begin{array}{lllllll}\text { Berechnet } ", & , & 10,05 & 20,08 & 30,09 & 25,06 & 30,04\end{array}$ $\begin{array}{llllllll}\text { Verbraucht } \quad " & 1 / 10 \mathrm{n}-\mathrm{Na}_{2} \mathrm{~S}_{2} \mathrm{O}_{3} & 15,09 & 30,13 & 45,16 & 37,60 & 45,08\end{array}$

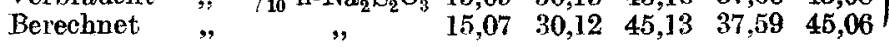

bei jedem Versuche waren $0,5 \mathrm{~g} \mathrm{NH}_{4} \mathrm{NO}_{3}$ anwesend

\section{Bemerkungen:}

Wie zu ersehen ist, wurden in allen Fällen genaue Werte erhalten. Die Jodatlösung war gegen die Thiosulfatlösung eingestellt. Hierbej. war zu berücksichtigen, daß $1 \mathrm{Mol} \mathrm{KJO}_{3}$ aus überschüssigem Kaliumjodid 6 Grammatome Jod frei macht, daß also der Oxydationswert des Jodats in Gegenwart ron Cyanwasserstoff $2 / 3$ des bei der Titerstellung mit Thiosulfat gefundenen beträgt. Bei der Titration des Jodcyanids mit Thiosulfat ist wiederum zu beachten, daß $1 / 3$ des Jodcyanids dem Jodat entstammt.

DerEndpunkt ist bei der Titration mit Jodat dann am schärfsten, wern in salzsaurer Lösung gearbeitet wird. ${ }^{1}$ ) Diese katalytische

1) Infolge der intermediăren Bildung von untertrijodiger Säure tritt gegen Ende der Reaktion zunächst ein rotvioletter Farbenton auf. Unmittelbar vor Erreichung des Fndpunktes muß daher gewartet werden, bis die Farbe der Jodstärke rein blau geworden ist, was wach einigen Bekunden stets der Fall ist. 
Wirkung von Chlorionen ist auf die Zwischenreaktion

$$
\mathrm{JO}_{3}{ }^{\prime}+5 \mathrm{Cl}^{\prime}+6 \mathrm{H}^{\prime}=\mathrm{JCl}+3 \mathrm{H}_{2} \mathrm{O}+2 \mathrm{Cl}_{2}
$$

zurückzuführen. Das naszierende Chlor entladet Jodion rascher als dies durch Jodat geschieht. In schwefelsaurer Lösung muß man gegen Ende der Titration, wenn die Flüssigkeit sich aufzuhellen beginnt, nach Zusatz eines jeden Tropfens 1-2 Minuten warten, da die Entfärbung langsamer erfolgt und man sonst leicht übertitriert. Wird aber der schwefelsauren Lösung Chlorid zugesetzt, so erfolgt der Farbenwechsel mit der gleichen Schärfe wie in chlorwasserstoffsaurer Lösung. Die Schärfe des Endpunktes ist ferner abhängig von der vorhandenen Wasserstoffionenkonzentration. Durch Versuche wurde festgestellt, daß in chlorwasserstoffsaurer Lösung zu Ende der Titration die Säurekonzentration wenigstens normal, in schwefelsaurer Lösung wenigstens doppeltnormal sein muß, wenn die möglichsto Schärfe des Endpunktes erzielt werden soll. Auf eine obere Grenze ler Säurekonzentration ist nicht besonders zu achten.

Bei Anwesenheit von Iromid färbt sich die Flüssigkeit gegen Ende der Oxydation infolge der Bildung von Jodbromid rotbraun. Bei geringerer Bromidkonzentration geht die rotbraune Farbe nach einigen Sekunden in die blaue der Jodstärke über. Bei größeren Bromidmengen dauert dieser Übergang mehrere Minuten. In einem solchen Falle braucht aber nicht abgewartet zu werden bis der Farbenton rein blau geworden ist, sondern man kann schon beim Eintreten einer violetten Farbennuance weitertitrieren. Der Endpunkt bleibt trotzdem scharf.

Zu einem bemerkenswerten Ergebnis gelangte ich bei der Titration mit Jodat hinsichtlich der Empfindlichkeit der Jodstärkereaktion. Ursprünglich war ich der Meinung, diese Mothode sei nur bei einem kleinen Reaktionsvolumen genau; denn wie bekannt, ist das Eintreten der Jodstärkereaktion an eine gewisse Jodidkonzentration gebunden. Da aber gegen Ende der Titration eben nur noch eine Spur Jodid vorhanden ist, glaubte ich, daß bei einem großen Reaktionsvolumen die Blaufärbung verschwinden müsse, ehe das Ende der Oxydation erreicht worden sei. Versuche zeigten aber, da $B$ dies vicht der Fall ist. So wurden $10 \mathrm{ccm}$ der Jodidlösung mit $8 \mathrm{ecm}$ Cyanid- und etwas Stärkelösung und schließlich mit $600 \mathrm{ccm} \mathrm{2,5fach}$ n-Salzsänure versetzt. Dann wurde mit der Jodatlösung titriert. Der Farbenumschlag erfolgte mit aller Schärfe bei dem theoretisch erforderlichen Jodatverbrauch von $10 \mathrm{cem}{ }^{1 / 40}$ mol-Jodatlösung. Wieder- 
holungen dieses Versuches auch beim Ansäuern mit $600 \mathrm{ccm}$ 5fach n-Schwefelsäure hatten das gleiche Ergebnis. Wurde nun nach beendeter Titration mit Jodat tropfenweise mit $1 / 10 \mathrm{n}$-Thiosulfatlösung versetzt, so zeigte sich an der Einfallstelle der einzelnen Tropfen Blaufärbung, die aber beim Umschütteln wieder verschwand. Erst wach Zusatz von 6-7 Tropfen zu der salzsauren oder 15-16 Tropfen zu der schwefelsauren Lösung blieb die Blaufärbung nach dem Umschütteln eben sichtbar bestehen. Bei einem Reaktionsvolumen von 100 ecm färbte der erste Tropfen Thiosulfat dauernd blau. Es ist somit klar, daß eine verschiedene Empfindlichkeit für das Eintreten und Verschwinden der Jodstärkereaktion besteht, da offenbar sehr verschiedene Jodidmengen für ihr Zustandekommen und für ihr Fortbestehen nötig sind. Die Titration mit Jodat ist daher anch hei einem großen Reaktionsvolumen genau.

In besonderen Versuchen wurde schließlich noch festgestellt, das Jodat auch bei stundenlanger Einwirkung auf Stärke weder in salzsarer noch in schwefelsaurer Jösung oxydierend wirkt.

\section{Methode $\mathrm{C}$.}

Die Lösung des Jodids wird mit dem gleichen Volumen minrestens aber mit $50 \mathrm{ccm} 2,5 \mathrm{fach}$ n-Salzsäure angesäuert, $6--8 \mathrm{~cm}$ $1 / 2 \mathrm{n}$-Kalinmeyanidlösung hinzugefügt und $1 / 10 \mathrm{n}$-Permanganatlösung bis zum fast völligen Furbloswerden der sich anfangs bräunenden Flüssigkeit zugetropft. Dann wird mit Stärkelösung versetzt und bis sum Verschwinden dex Blaufärbung titriert. Das gebildete Jodeyanid kann jetot wieder mit Thiosulfat titriert werden. Chloride und Nitrate stören nicht, dagegen dürfen Bromide nicht anwesend seim.

Beleganalysen:

\begin{tabular}{|c|c|c|c|c|c|c|c|c|}
\hline \multirow{4}{*}{$\begin{array}{l}\text { Angewendet } \\
\text { Verbraucht } \\
\text { Berechnet }\end{array}$} & \multirow{4}{*}{$\begin{array}{l}\mathrm{ccm} \\
, " \\
, \\
,\end{array}$} & \multirow{4}{*}{$\begin{array}{l}\mathrm{K}+\mathrm{J} \\
1 / 10 \mathrm{n}-\mathrm{KMnO} \mathrm{O}_{4} \\
1 / 10 \mathrm{n}-\mathrm{Na}_{2} \mathrm{~S}_{2} \mathrm{O}_{3} \\
1 / 10 \mathrm{n}-\mathrm{KMnO}_{4} \\
\text { oder: } \mathrm{Na}_{2} \mathrm{~S}_{2} \mathrm{O}_{3}\end{array}$} & \multirow{3}{*}{$\begin{array}{l}10,04 \\
10,06 \\
10,07\end{array}$} & \multirow{3}{*}{$\begin{array}{l}20,1 \\
20,13 \\
20,13\end{array}$} & \multirow{3}{*}{$\begin{array}{l}25,08 \\
25,08 \\
25,09\end{array}$} & \multirow{3}{*}{$\begin{array}{l}30,05 \\
30,06 \\
30,07\end{array}$} & \multirow{3}{*}{$\begin{array}{l}40,16 \\
40,13 \\
40,15\end{array}$} & \multirow{3}{*}{$\begin{array}{l}45,12 \\
45,12 \\
45,14\end{array}$} \\
\hline & & & & & & & & \\
\hline & & & & & & & & \\
\hline & & & 10,04 & 20,10 & 25,08 & 30,04 & 40,15 & 45,11 \\
\hline $\begin{array}{l}\text { Augewendet } \\
\text { Verbraucht } \\
\text { Berechnet }\end{array}$ & $\begin{array}{c}\text { sem } \\
",\end{array}$ & $\underset{1 / 10}{\mathrm{~K}} \mathrm{\textrm {I }}-\dot{\mathrm{K}}_{\|} \dot{\mathrm{MnO}} \dot{\mathrm{O}}_{4}$ & $\begin{array}{l}10,06 \\
11,58 \\
10,06\end{array}$ & $\begin{array}{l}20,04 \\
21,89 \\
20,04\end{array}$ & $\begin{array}{l}25,12 \\
27,05 \\
25,12\end{array}$ & $\begin{array}{l}30,09 \\
31,93 \\
30,08\end{array}$ & & $\begin{array}{l}3 r \text { a } \\
\text { nd }\end{array}$ \\
\hline
\end{tabular}

\section{Bemerkungen:}

Bei der Titration mit Permanganat darf die Stärke erst gegen Ende der Oxydation zugefügt werden, da sonst das Permanganat 
merklich auf die Stärke einwirkt, namentlich wenn viel Manganosalz anwesend ist. So wurden für $10,05 \mathrm{cem}$ der Jodidlösung bei sofortigem Stärkezusatz $10,07 \mathrm{~cm} 1 / 10 \mathrm{n}-\mathrm{KMnO}_{4}$ in schwefelsaurer Lösung und für $10,03 \mathrm{cem} \mathrm{KJ}$ bei Zusatz von Stärke und viel Mangansulfat $10,11 \mathrm{ecm} 1 / 10 \mathrm{n}-\mathrm{KMnO}_{4}$ verbraucht. Ferner wurden für $40 \mathrm{ccm} \mathrm{KJ}$ bei sofortigem Stärkezusatz $40,12 \mathrm{ccm} 1 / 10 \mathrm{n}-\mathrm{KMnO}_{4}$ in salzsaurer und $40,29 \mathrm{ccm}$ in schwefelsaurer Lösung statt der erforderlichen $39,99 \mathrm{ccm}$ verbraucht. Diese Versuche zeigen, daß bei geringen Jodidmengen ein sofortiger Stärkezusatz praktisch keine Rolle spielt, wenn Manganosalz abwesend ist. Bei größeren Jodidmengen macht sich schon die Einwirkung des Permanganats auf die Stärke geltend, und zwar in schwefelsaurer Lösung mehr als in salzsaurer. Man arbeitet daher am besten in salzsaurer Lösung und setzt die Stärke erst knapp vor Beendigung der Titration zu.

Die Anwesenheit von Bromiden erhöht den Permanganatverbrauch bedeutend. Die Reaktion zwischen Bromid und Permanganat verläuft so rasch, daB schon Bromion entladen wird, wenn noch nicht alles Jodid verbraucht ist. Das frei gewordene Brom reagiert dann mit dem Cyanwasserstoff unter Bromcyanbildung weiter. Bromeyan ist aber ein sehr schwaches Oxydationsmittel, das nur träge auf noch vorhandenes Jodid unter Regeneration von Bromid einwirkt. Es wird daher schließlich am Ende der I'itration nebst dem Jodid auch ein Teil des Bromids oxydiert worden sein.

MAC Curio $\mathrm{CH}^{x}$ ) hat versucht Jodide in Gegenwart von Cyanwasserstoff in der Art zu bestimmen, daß er in schwefelsauerer Lüsung mit Permanganat bis zur bleibenden Rosafärbung titrierte. Doch werden so etwas zu hohe Werte erhalten. Nach dieser Methode wurden z. B. für $25,10,30,08,40,12 \mathrm{ccm}$ der Jodidlösung 25,21, 30,17 und $40,26 \mathrm{~cm} 1 / 10 \mathrm{n}-\mathrm{KMnO}_{4}$ statt der berechneten 25,10, 30,07, $40,11 \mathrm{ccm}$ verbraucht. Titrationen mit Permanganat bis zur Rosafärbung bei Anwesenheit von Cyanwasserstoff werden im allgemeinen. zu hohe Werte exgeben. So verbrauchte ich für $26,12,30,09,40,20 \mathrm{ccm}$. einer $1 / 10 \mathrm{n}$-Ferrosulfatlösung in Gegenwart Schwefelsäure und Cyanwasserstoff $26,22,30,21$ and $40,35 \mathrm{~cm} 1 / 10 \mathrm{n}-\mathrm{KMnO}_{4}$. Wahrscheinlich begünstigt Cyanwaserstoff die Bildung von Manganisalz, so daß nicht sofort der geringste Überschuß von Permanganat zu erkennen ist.

Was die Qualität des bei den beschriebenen Methoden zu verwendenden Kaliumeyanids anbelangt, ist $z u$ fordern, daB dasselbe

1) Chem. News 67 (1888), 135. 
frei von Ferrocyanid sei. $10 \mathrm{ccm}$ der $1 / 2 \mathrm{n}$. mit Schwefelsäure angesäuerten Cyanidlösung sollen mit 1 Tropfen einer $1 / 10$ n-Permanganatlösung dauernde Rosafärbung geben. Dies ist bei einem KaHLbaumschen Präparat "Zur Analyse" der Fall, das auch bei allen Versuchen verwendet würde.

$\mathrm{Ob}$ nach den angegebenen Methoden auch Jod in unlöslichen Jodiden bestimmt werden kann, soll einer besonderen Untersuchung vorbehalten bleiben.

\section{Bestimmung zweier 0xydationsstufen des Jods nebeneinander.}

a) Bestimmung von Jodid neben freiem Jod.

Man löst in $10 \mathrm{~cm} 1 / 2 \mathrm{n}$-Kaliumcyanidlösung, versetzt mit $50 \mathrm{ccm} 2,5$ fach n-Salzsäure, fügt etwas Stärkelösung hinzu und titriert zuerst mit $1 / 40 \mathrm{~mol}$. Jodat- und dann mit $1 / 10 \mathrm{n}$-Thiosulfatlösung.

\begin{tabular}{|c|c|c|c|c|c|c|}
\hline \multirow[b]{2}{*}{ Angewendet } & \multicolumn{6}{|c|}{ Beleganalysen: } \\
\hline & ecm & $1 / 2 \xi n-K J$. & 10,02 & 15,06 & 20,12 & 20,03 \\
\hline & ", & $\left.1 / 10 n-J^{1}\right\}$ & 10,10 & 10,08 & 5,05 & 5,07 \\
\hline Verbraucht & ," & $1 / 40 \mathrm{~mol}^{2} \mathrm{KJO}_{3}$ & 20,13 & 25,17 & 25,19 & 25,10 \\
\hline Berechnet & " & & 20,12 & 25,14 & 25,17 & 25,10 \\
\hline Verbraucht & $"$ & $1 / 10 \mathrm{n}-\mathrm{Na}_{2} \mathrm{~S}_{2} \mathrm{O}_{3}$ & 40,30 & 47,81 & 42,83 & 42,84 \\
\hline Berechnet & " & ", & 40,28 & 47,79 & 42,80 & 42,82 \\
\hline
\end{tabular}

Bezeichnet man mit $m$ die Anzahl der verbrauchten Kubikzentimeter $1 / 40$ mol. Jodat-, mit $n$ die der $1 / 10$ n-Thiosulfatlösung, so berechnet man das gebundene Jod nach

und das freie Jod nach

$$
J_{-1}=\left(\frac{5}{2} m-n\right) \cdot \frac{J}{2 \cdot 10^{4}} \mathrm{~g}
$$

$$
J_{0}=\left(m-\frac{3}{2} n\right) \frac{J}{10^{4}} g .
$$

b) Bestimmung von freiem Jod neben positir einwertigem Jod.

Man verfährt wie bei a).

\begin{tabular}{|c|c|c|c|c|c|c|}
\hline Angewendet & $\mathrm{com}$ & $\left.1 / 10 n-J^{1}\right\rangle$. & 5 & 5,03 & 10,04 & 15,12 \\
\hline & & $1 / 20 \mathrm{~mol} . \mathrm{JCN}$ & 25,1 & 20,02 & 15,05 & 10,09 \\
\hline Verbraucht & ", & $1 / 40 \mathrm{~mol} \mathrm{KJO}_{3}$ & 5,02 & 5,03 & 10,06 & 15,12 \\
\hline Berechnet & ," & & 5 & 5,03 & 10,04 & 15,12 \\
\hline Verbraucht & $"$ & $1 / 10 \mathrm{n} \cdot \mathrm{N} \mathrm{a}_{2} \mathrm{~S}_{2} \mathrm{O}_{3}$ & 37,63 & 32,60 & 40,18 & 47,90 \\
\hline Berechnet & " & , & 37,60 & 32,59 & 40,15 & 47,89 \\
\hline
\end{tabular}

$$
\text { Beleganalysen: }
$$

1) Es wurde cyankalische Jodlösung, im Liter $1 / 20$ Mol $\mathrm{J}_{2}$ und $1 / 20$ Mol KCN enthaltend, angewendet. 
Werden $m$ eem der Jodat- und $n$ eem der Thiosulfatlösung verbraucht, so berechnet man das freie Jod nach

$$
J_{0}=m \cdot \frac{J}{10^{\mathrm{x}}} \mathrm{g}
$$

und das positiv einwertige Jod nach

$$
J_{+1}=\left(n-\frac{5}{2} m\right) \cdot \frac{\pi}{2 \cdot 10^{4}} \mathrm{~g} .
$$

c) Bestimmung ron freiem Jod neben Jodatjod.

Man löst in $10 \mathrm{ccm} 1 / 2 \mathrm{n}$-Kaliumcyanidlösung, säuert mit $50 \mathrm{ccm}$ 2,5fach n-Salzsäure an, fügt etwas Stärkelösung hinzu und titriert mit einer $1 / 10 \mathrm{n}$-Zinnchlorürlösung bis zum Verschwinden der Jodstärkereaktion, die erst im Verlaufe der Titration eintritt, wenn Jodat im Überschuß vorhanden war. Dann titriert man mit 1/40 mol. Jodatlösung. Die Zinnchlorürlösung muß unmittelbar vor der Analyse gegen die Jodatlösung eingestellt werden.

\begin{tabular}{|c|c|c|c|c|c|c|}
\hline Angewende & $\mathrm{ccm}$ & $\left.1 / 10 \mathrm{n}-J^{1}\right)$. & 5,02 & 10,04 & 15,04 & 20,10 \\
\hline & , & $\mathrm{l} / 40 \mathrm{~mol}^{\mathrm{KJJO}}$ & 25,08 & 20,05 & 15,06 & 10,00 \\
\hline $\begin{array}{l}\text { Verbraucht } \\
\text { Berechnet }\end{array}$ & $"$ & $1 / 10 \mathrm{n}-\mathrm{SnCl}_{2}$. & $\begin{array}{l}42,67 \\
42,64\end{array}$ & $\begin{array}{r}40,13 \\
40,11\end{array}$ & $\begin{array}{l}37,65 \\
37,63\end{array}$ & $\begin{array}{l}35,18 \\
35,17\end{array}$ \\
\hline Verbraucht & $"$ & $1 / 40 \mathrm{~mol}^{1 . \mathrm{KJO}} \mathrm{JO}_{3}$ & $\begin{array}{l}72,07 \\
22,60\end{array}$ & 30,11 & 37,64 & 45,23 \\
\hline Berechnet & & & 22,58 & 30,10 & 37,61 & 45,22 \\
\hline
\end{tabular}

Beleganalysen:

Wurden $m$ ccm Zinnchlorür- und $n$ cem Jodatlösung verbrancht, so berechnet man das freie Jod nach

und das Jodatjod nach

$$
\digamma_{0}=\left(\frac{3}{5} n-\frac{1}{5} m\right) \cdot \frac{J}{10^{4}} \mathrm{~g}
$$

$$
J_{+5}=\left(\frac{6}{5} m-\frac{3}{5} \cdot n\right) \cdot \frac{J}{6 \cdot \therefore 10^{4}} \mathrm{~g} .
$$

\section{Titration von Jodat mit Jodid.}

Wie man Jodid mit Jodat, so kann man auch umgekehrt Jodat mit Jodid in Gegenwart von Cyanwasserstoff titrieren. Die Erscheinungen sind verschieden, je nachdem man in salzsaurer oder schwefelsaurer Lösung arbeitet.

Titriert man eine chlorwasserstoffsaure Lösung von Jodat in Gegenwart von Cyanwasserstoff und Stärke mit $1 / 20$ n-Jodidlösung,

1) Vgl. Anmerkung 1 auf S. 340 . 
so erhält man keinen scharfen Endpunkt. Beim jedesmaligen $\mathrm{Zu}$ tropfen des Jodids entsteht die charakteristische dunkelbraune Färbung von untertrijodiger Säure ${ }^{1}$, die bald der hellgelben von Jodmonochlorid Platz macht. Hat man die theoretisch erforderliche Menge Jodid zugesetzt, um alles Jodat zu positiv einwertigem Jod zu reduzieren, so ruft ein weiterer Jodidzusatz eine allmählich intensiver werdende, dauernde Braunfärbung hervor, die bei einem U̇berschuß von 1-2 ecm der Jodidlösung rasch in die Farbe der Jodstärke übergeht. Titriert man jetzt diese überschüssige Menge Jodid wieder mit Jodat bis zum Verschwinden der Jodstärkereaktion, so erhält man wieder einen scharfen Endpunkt und eine farblose Lösung von Jodcyanid. Eine solche erhält man auch aus Jodmonochlorid bei längerer Einwirkung von Cyanwasserstoff.

In schwefelsaurer Lösung läßt sich Jodat in Gegenwart von Cyanwasserstoff direkt mit Jodid titrieren, wenn das Realitionsvolumen nicht zu groß ist. Solange noch unverbrauchtes Jodad vorhanden ist, wird die Flüssigkeit immer wieder farblos, da alles Jodid zu Jodideyanid oxydiert und die äquivalente Menge Jodat zu Jodcyanid reduziert wurde. Der erste überschüssige Tropfen der Jodidlösung ruft dauernde Blaufärbung hervor, indem nach der Reaktion

Jod frei wird.

$$
\mathrm{JCN}+\mathrm{H}+\mathrm{J}^{\prime} \rightarrow \mathrm{J}_{2}+\mathrm{HCN}
$$

Die Erklärung, weshalb bei der Titration von Jodid mit Jodat in salzsaurer Lösung bei Anwesenheit von Cyanwasserstoff ein scharfer Endpunkt und nur Jodcyanid als Endpunkt erhalten wird, während bei der umgekehrten Titration Jodmonochlorid auftritt und kein scharfer Endpunkt zu erzielen ist, ist folgende: Wird Jodid mit Jodat titriert, so werden alle höheren Jodverbindungen, die als Zwischenstufen gebildet werden, bis zu Jod reduziert, da bis zu Ende der Reaktion zufolge der Umsetzung

$$
\mathrm{J}_{2}+\mathrm{HCN} \rightarrow \mathrm{JCN}+\mathrm{H}^{*}+\mathrm{J}^{\prime}
$$

Jodid vorhanden ist. Auch intermediär gebildetes Jodmonochlorid wird sich stets mit Jodid zu freiem Jod umsetzen, welches dann wieder mit Cyanwasserstoff weiterreagiert, so daß als Endprodukt nur Jodcyanid erhalten werden kann. Im umgekehrten Falle, wo also Jodat im Überschuß zugegen ist, geht die Oxydation des Jodids in salzsaurer Lösung sofort quantitativ bis zu positiv einwertigem Jod,

1) Über untertrijodige Säure vgl. SKRABAL und BUCHTA, Chemikerzeitung $\mathbf{8 R}$ (1909), 1184, 1193. 
welches zunächst als Jodmonochlorid stabilisiert bleibt, um ganz langsam in Jodcyanid überzugehen, wie noch genauer im nächsten Abschnitt auszuführen sein wird. In Gegenwart von Jodmonochlorid ist aber bei der Titration mit Jodid kein Indpunkt zu erzielen, denn der erste überschüssige Tropfen reagiert nach dem Sehema:

$$
\mathrm{J}^{\prime}+2 \mathrm{JCl}+\mathrm{H}_{2} \mathrm{O}=\mathrm{J}_{3} \mathrm{OH}+\mathrm{H}^{\prime}+2 \mathrm{Cl}^{\prime}
$$

unter Bildung von untertrijodiger Säure, die keine Jodstärkereaktion gibt. Untertrijodige Säure reagiert zwar mit Cyanwasserstoff unter Jod- and Jodidbildung weiter, doch wird erstere wegen des überschüssig vorhandenen Jodmonochlorids immer wieder regeneriert, so daß erst nach längerer Zeit, wenn schon alles Jodmonochlorid umgesetzt ist, die Jodstärkereaktion eintreten kann. Rasch tritt letztere ein, wenn ein größerer Überschuß von Jodid zugesetzt wird. Hat man also Jodat in salzsaurer liösung bei Gegenwart von Cyanwasserstoff mit Jodid zu titrieren, wie das bei Restmethoden der Fall ist, so wird man praktisch so verfahren, daß man die Jodidlösung bis zum Auftreten der Blaufärbung zufließen läßt und dann mit der Jodatlösung zu Ende titriert.

Während aber bei der Titration von Jodid mit Jodat die Konzentration der Salzsäure nach oben hin fast beliebig geändert werden kann, ist dies bei der umgekehrten Titration durchaus nicht der Fall. Man wird hier am besten die Konzentration der Salzsäure nicht über $2 \mathrm{n}$. hinausgehen lassen. Bei dieser Konzentration wird Jodat noch nicht freiwillig durch Salzsäure zersetzt.1) In konzentriert salzsaurer Lösung findet die Umsetzung

$$
\mathrm{JO}_{3}{ }^{\prime}+5 \mathrm{Cl}^{\prime}+6 \mathrm{H}^{\prime}=\mathrm{JCl}_{3}+3 \mathrm{H}_{2} \mathrm{O}+\mathrm{Cl}_{2}
$$

statt. Ist nun Cyanwasserstoff anwesend, so bildet das entbundene Chlor sofort Chlorcyan, welches auf Jodid von nur sehr geringer Oxydationswirkung ist. Es würde daher bei der Titration viel zu wenig Jodid verbraucht werden. Dies wird in folgenden Versuchen gezeigt:

1. $100 \mathrm{ccm}$ Chlorwasser schieden aus Jodkalium Jod aus, zu dessen Reduktion $15,3 \mathrm{ccm} 1 / 10 \mathrm{n}$-Thiosulfat nötig waren. Wurde aber in wiederholten Versuchen die gleiche Menge Chlorwasser zuerst

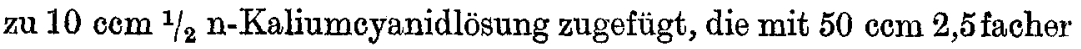

1) Nach einer alten Angabe BunsteNs (Lieb. Ann. 86 (1853), 265) wird Jodat schon durch n.-Salzsäure quantitativ zersetzt. Dem widerspricht das Ergebnis der oben weiterhin angeführten Versuche. 
Salzsäure angesäuert war, und dann mit Jodkalium versetgt, so wurden bei der Titration nur $0,15-0,2 \mathrm{ccm}$ Thiosulfat verbraucht, ein Beweis, dab Chlorcyan ein nur sehr wenig wirksames Oxydationsmittel ist.

2. $30 \mathrm{ccm} \mathrm{1/40} \mathrm{mol.} \mathrm{Jodatlösung} \mathrm{warden} \mathrm{mit} 8 \mathrm{ccm} \mathrm{1/2} \mathrm{n}$-Cyanidlösung versetzt, Stärke hinzugefügt and mit $50 \mathrm{ccm} 2,5 \mathrm{fach} \mathrm{n-Salz-}$ säure angesäuert. Dann wurde mit $1 / 20 \mathrm{n}$-Jodidlösung wie oben angegeben, titriert. Für insgesamt $30,91 \mathrm{ccm} 1 / 40 \mathrm{~mol}$. Jodat- wurden $30,90 \mathrm{ccm} 1 / 20 \mathrm{n}$-Jodidlösung verbraucht. Der Versuch wurde nun derart wiederholt, daß das Flüssigkeitsgemisch vor der Titration im verschlossenen Kolben 16 Stunden stehen blieb. Es wurden so für $31,25 \mathrm{ccm} \mathrm{1/40}$ mol. Jodat- $31,22 \mathrm{~cm} 1 / 20 \mathrm{n}$-Jodidlösung verbraucht. In beiden Fällen war also der. Jodidverbrauch der Theorie entsprechend. Wurden aber zum Ansäuern $40 \mathrm{~cm}$ konzentrierte Salzsäure $(1,196)$ :ngewendet, so färbte sich das Lösungsgemisch gelb und bei der Titration mit Jodid wurden für insgesamt $36,61 \mathrm{cem} 1 / 40$ mol. Jodat- nur $20,92 \mathrm{cem} 1 / 20 \mathrm{n}$-Jodidlösung verbraucht. Es hatte also hier weitgehend Chlorcyanbildung stattgefunden.

\section{v. Verhalten von Cyanwasserstoff gegen Jodmonoohlorid and untertrijodige Säure.}

Um über das Verhalten von Cyanwasserstoff gegen Jodmonochlorid Klarheit zu erlangen, wurden folgende Versuche angestellt:

$Z u$ je $10 \mathrm{cem} 1 / 20$ n-Jodidlösung wurde immer $10 \mathrm{ccm} 1 / 10 \mathrm{n}$-Permanganatlösung, also genau die Menge, die nötig ist, um alles Jodid zu Jodmonochlorid zu oxydieren, hinzugefügt. Dann wurde in vier Fällen mit je $50 \mathrm{ccm} 2,5 \mathrm{n}$. und in einem 5 . Falle mit $50 \mathrm{ccm}$ konzentrierter Salzsäure $(1,196)$ versetzt. Die erhaltene hellgelbe Lösung von Jodmonochlorid wurde im 1. Falle mit Thiosulfat ohne Zusatz von Jodkalium bis zum Verschwinden der Jodstärkereaktion titriert. Verbraucht wurden $7,42 \mathrm{~cm} \quad 1 / 10 n-\mathrm{Na}_{2} \mathrm{~S}_{2} \mathrm{O}_{3}$-Lösung. Im 2. Falle wurden $15 \mathrm{~g}$ Natriumacetat und dann $5 \mathrm{ccm} 1 / 2 \mathrm{n}$-Kaliumcyanidlösung hinzugefügt; der Zusatz von Cyanid bewirkte eine augenblickliche Entfärbung der Flüssigkeit. Die hierauf vorgenommene Titration mit Thiosulfat ergab einen Verbrauch von $10,02 \mathrm{ccm}$ $1 / 10 \mathrm{n}$-Lösung. Im 3. Falle wurden $10 \mathrm{ccm} \mathrm{1/2} \mathrm{n}$-Kaliumcyanidlösung allein hinzugefügt und nach 15 Minuten - erst nach dieser Zeit war hier vollständige Entfärbung eingetreten - titriert. Verbrauch: $10,01 \mathrm{ccm} 1 / 10 \mathrm{n}-\mathrm{Na}_{2} \mathrm{~S}_{2} \mathrm{O}_{3}$-Lösung. Im 4. Falle wurde mit $20 \mathrm{ccm}$ $1 / 2$ n-Kaliumcyanidlösung versetzt. Die Entfärbung der Flüssigkeit trat nach 3 Minuten ein. Verbrauch bei der Titration: 10,01 ccm 
$1 / 10 \mathrm{n}-\mathrm{Na}_{2} \mathrm{~S}_{2} \mathrm{O}_{3}$-Lösung. Im 5. Falle wurden wieder $20 \mathrm{ccm} \mathrm{1/2} \mathrm{n-Ka-}$ liumcyanidlösung zugefügt. Hier entfärbte sich die Lösung erst nach 12 Stunden vollständig. Bei der dann vorgenommenen Titration ergab sich ein Verbrauch von $10,03 \mathrm{ccm} 1 / 10 \mathrm{n}-\mathrm{Na}_{2} \mathrm{~S}_{2} \mathrm{O}_{3}$-Lösung.

Beim ersten Versuche wurden ungefähr nur $s / 4$ der Thiosulfatmenge verbraucht, die notwendig ist, um Jodmonochlorid unter Jodidzusatz zu titrieren, da die Jodkationen ${ }^{1}$ ) enthaltende Lösung von Jodmonochlorid das Thiosulfat teilweise bis zu Sulfat oxydiert. Bei den übrigen Versuchen aber war der Thiosulfatverbrauch stets ein solcher, wie er erforderlich ist, um Thiosulfat quantitativ zu Tetrathionat zu oxydieren, ein Beweis, daß hier das Jodmonochlorid vollständig in Jodcyanid übergegangen war und worauf auch aus der Entfärbung der Flüssigkeit geschlossen werden konnte. Die Versuche zeigen auch deutlich, daß die Geschwindigkeit der Reaktion

$$
\mathrm{JCl} \longrightarrow \mathrm{JCN}
$$

außer von der Massenwirkung des Cyanwasserstoffes auch von der Wasserstoffionenkonzentration in dem Sinne abhängig ist, da mit wachsender Azidität die Gesehwindigkeit des Vorganges abnimmt. Diese Verhältnisse lassen sich erklären, wenn man die verschiedenen Dissoziation von Jodmonochlorid und Jodeyanid in Betracht zieht. Ersteres ist in wäßriger Lösung elektrolytisch nach

und hydrolytisch nach

$$
\mathrm{JCl} \rightleftharpoons \mathrm{J}^{\prime}+\mathrm{Cl}^{\prime}
$$

$$
\mathrm{JCl}+\mathrm{H}_{2} \mathrm{O} \rightleftharpoons \mathrm{JOH}+\mathrm{HCl}
$$

dissoziiert.1) Dagegen ist Jodeyanid als elektrolytisch und hydrolytisch fast undissoziliert anzusehen. Hierfür sprechen seine Titrierbarkeit mit Thiosulfat, zum Beweise, daß in seiner Lösung weder Jodkationen noch ihr Hydrolysenprodukt, unterjodige Säure vorhanden sind, sowie seine Nichtfällbarkeit mit Silbernitrat, zum Beweise, da $B$ es gelöst weniger $\mathrm{CN}^{\prime}$-Ionen abspaltet als freier Cyanwasserstoff, der durch Silberion sofort gefällt wird. Treffen Jodkationen mit Cyanwasserstoff zusammen, so wird solange Jodcyanidbildung erfolgen, als mit dem beinahe ganz links liegenden Gleichgewicht

$$
\mathrm{JCN} \rightleftharpoons \mathrm{J}+\mathrm{CN}^{\prime}
$$

verträglich ist. Praktisch wird dies also eine quantitative Jodeyanidbildung bedeuten. Das Jodmonochlorid wird nun bei mittleren Salz-

1) Vgl. ABEga, Handbuch d. anorg. Chemie IV, 2, 8. 465. 
säurekonzentrationen relativ am meisten Jodkationen abspalten, in konzentrierter Salzøäure erfolgt Komplexbildung nach

$$
\mathrm{JCl}+\mathrm{HCl} \rightleftharpoons \mathrm{JCl} \cdot \mathrm{HCl}^{1} \text { ) }
$$

and daher Abnahme der J-Ionenkonzentration und nur hier wird Jodmonochlorid neben Cyanwasserstoff längere Zeit bestehen können. ${ }^{2}$ ) Tatsächlich erfolgte die Reaktion

$$
\mathrm{JCl}+\mathrm{HCN}=\mathrm{JCN}+\mathrm{HCl}
$$

bei Versuch 4 ungleich viel raseher als bei Versuch 5. Zudem aber kommt bei abnehmender Salzsäurekonzentration die Zunahme der hydrolytischen Spaltung des Jodmonochlorids und man sieht bei Versuch 2, der durch geringste Säurekonzentration und dementsprechend am weitesten fortgeschrittene Hydrolyse ausgezeichnet ist, die Jodcyanidbildung trotz geringster Cyanwasserstoffkonzentration am raschesten erfolgen. Es wird also auch das Hydrolysenprodukt des Jodmonochlorids, die unterjodige Säure, mit Cyanwasserstoff unter Jodcyanidbildung reagieren. ${ }^{3}$ ) Die Reaktion wird als Salzbildung im Sinne der Gleichung

$$
\mathrm{JOH}+\mathrm{HCN}=\mathrm{JCN}+\mathrm{H}_{2} \mathrm{O}
$$

aufzufassen sein, wobei das positiv einwertige Jod der nur wenig beständigen unterjodigen Säure stabilisiert wird.

Einfach läßt sich auch die Einwirkung von Cyanwasserstoff auf untertrijodige Säure erklären. Läßt man auf eine schwefelsaure Jodidlösung Jodat in solcher Menge einwirken, daß wohl alles Jod frei gemacht wird, jedoch nicht unter entsprechenden Bedingungen ganz zu positiv einwertigem Jod oxydiert werden könnte, so erhält man eine braune Lösung von untertrijodiger Säure, die sich auf Stärke-

${ }^{1}$ Skabal und Buchta, Chemikerzeitung 333 (1909), 1184, 1193; Sullivan, Z. physik. Chem. 28 (1899), 523.

2) ABEgas Handbuob IV, 2, S. 479 fíhrt unter Berufung auf E. v. MEYer (Journ. prakt. Chem. 36 (1887), 292) an, daß hinreichend konzentrierte Salzsäure Jodeyanid nach: $\mathrm{JCN}+\mathrm{HCl} \rightarrow \mathrm{JCI}=\mathrm{HCN}$ zersetzt. Ich fand in der Originalarbeit Meynes keine derartige Angabe. Eine solche Zersetzung wäre aber der entgegengesetzte Vorgang des oben festgestellten Überganges von Jodmonochlorid in Jodcyanid. Vielleicht besteht in konzentriert salzsaurer Lösung das Gleichgewicht $\mathrm{JCN}+2 \mathrm{HCl} \rightleftharpoons \mathrm{JCl} \cdot \mathrm{HCl}+\mathrm{HCN}$. Schon eine mäBige Verdtinnung (wie im obigen Versuch 5) wird den Komplex $\mathrm{JCl} \cdot \mathrm{HCl}$ so weit zersetzen, daB sich quantitativ Jodcyanid bildet.

3) In alkaliseher Lösung, also auf $\mathrm{CN}^{\prime}$-Ion wirkt unterjodige Säure unter Cyanatbildung narh $\mathrm{JOH}+\mathrm{CN}^{\prime}+\mathrm{OH}^{\prime}=\mathrm{J}^{\prime}+\mathrm{CNO}^{\prime}+\mathrm{H}_{2} \mathrm{O}$ ein. RuPP, Arch. Pharm. 243 (1905), 458. 
zusatz rotbraun färbt. Fügt man nun $5 \mathrm{ccm} 1 / 2 \mathrm{n}$-Kaliumcyanidlösung hinzu, so verschwindet die braune Farbe nach einigen Sekunden und macht der schön blauen der Jodstärke Platz. Unterjodige Säure spaltet nämlich Jod ab nach der Gleichgewichtsbeziehung

$$
\left.\mathrm{J}_{3} \mathrm{OH} \rightleftharpoons \mathrm{JOH}+\mathrm{J}_{2} \cdot{ }^{1}\right)
$$

Tritt nun Cyanwasserstoff hinzu, so wird dieses Gleichgewicht sofort durch die hintereinander bzw. nebeneinander verlaufenden Reaktionen:

$$
\begin{aligned}
& \mathrm{J}_{2}+\mathrm{HCN} \rightarrow \mathrm{JCN}+\mathrm{H}^{\cdot}+\mathrm{J}^{\prime} \\
& \mathrm{H}^{\prime}+\mathrm{J}^{\prime}+\mathrm{JOH}=\mathrm{J}_{2}+\mathrm{H}_{2} \mathrm{O} \\
& \mathrm{J}_{2}+\mathrm{HCN} \rightarrow \mathrm{JCN}+\mathrm{H}^{\prime}+\mathrm{J}^{\prime}
\end{aligned}
$$

gestört, so daß sich als Bruttovorgang die Summe von (1), (2), (3) und (4) ergibt:

$$
\mathrm{J}_{3} \mathrm{OH}+2 \mathrm{HCN}=2 \mathrm{JCN}+\mathrm{H} .+\mathrm{J}^{\prime}+\mathrm{H}_{2} \mathrm{O} .
$$

Jodcyanid aber gibt mit Jodid die Jodstärkereaktion.

Die bei der Titration von Jodat mit Jodid in Gegenwart von Cyanwasserstoff gemachten Beobachtungen lassen erkennen, daB die Stabilisierung des positiv einwertigen Jods im Sinne des Schemas

$$
\mathrm{JOH} \rightarrow \mathrm{JCl} \rightarrow \mathrm{JCN}
$$

vor sich geht. Und zwar erfolgt der Utbergang

$$
\mathrm{JOH} \rightarrow \mathrm{JCl}
$$

rasch, während JCl nur langsam mit HCN reagiert. Fehlt der Chlorwasserstoff, wio bei der Titration in schwefelsaurer Lösung, dann geht JOH direkt, und zwar rasch jin JCN über. Dieses Verhalten entspricht den Forderungen der Reaktionsstufenregel. Zunächst tritt das positiv einwertige Jod in seiner labilsten Form, der unterjodigen Säure, auf. Der Utbergang in das schon sehr stabile Jodmonochlorid oder in das noch beständigere Jodeyanid exfolgt rasch, während der Übergang von der einen stabilen Form in die andere sehr langsam vor sich geht. Da aber die Stabilität von Jodmonochlorid in wäBriger Lösung, wie es scheint, nur von seinem jeweiligen Dissoziationszustand abhängig ist, wäre es möglich, daß in konzentriert chlorwasserstoffsaurer Lösung auch die Reaktionsfolge

$$
\mathrm{JOH} \rightarrow \mathrm{JCN} \rightarrow \mathrm{JCl}
$$

stattfindet.2) Hier hätte der Versuch zu entscheiden.

1) Skrabat, Monatshefte f. Chemie 32 (1911), 815.

2) Vgl. Anmerkung 2, S. 346 . 


\section{Zusammenfassung.}

1. Es wurden volumetrische Methoden zur Bestimmung von Jodid angegeben, die darauf beruhen, daß Jodid in salz- oder schwefelsaurer Lösung bei Anwesenheit von Cyanwasserstoff durch oxydierende Maßflüssigkeiten (Jodat und Permanganat) zu Jodcyanid oxydiert wird, wobei das Verschwinden der Jodstärkereaktion den Endpunkt anzeigt. An diese Oxydation kann unmittelbar die Messung des gebildeten Jodcyanids mit Thiosulfat angeschlossen werden, so daB jede Bestimmung in doppelter Art durchführbar ist. Auch Nitrit kann als Oxydationsmittel angewendet werden; die Bestimmung geschieht dann mit Thiosulfat allein, nachdem der Überschuß des Nitrits mit Harnstoff zerstört wurde.

2. Die Brauchbarkeit der Methoden in Gegenwart von Bromid und Nitrat wurde geprüft.

3. Es wurde an einigen Beispielen gezeigt, wie verschiedene Oxydationsstufen des Jods nebeneinander bestimmt werden können.

4. Es wurde geprüft, unter welchen Bedingungen auch Jodat mit Jodid in Gegenwart von Cyanwasserstoff titriert werden kann.

5. Es wurde das Verhalten von Cyanwasserstoff gegen Jodmonochlorid und unterjodige Säure untersucht.

Brünn, Laboratorium für anorganische, physikalische und anaiytische Chemie der Deutschen Technischen Bochschule.

Bei der Redaktion eingegangen am 11. April 1922. 